\title{
Electron Microscopic Basis for Applications of Immunohistochemistry (IHC) and in situ Hybridization (ISH) to Diagnostic Pathology
}

\author{
R. Yoshiyuki Osamura \\ Department of Pathology, Tokai University School of Medicine, Boseidai Isehara-city, Kanagawa 259-112
}

Key words: Electron microscopy, Immunohistochemistry, In situ hydridization, Diagnostic pathology, Immunoelectron microscopy

\section{Introduction}

Immunohistochemistry (IHC) to detect tissue antigens by antibodies has been applied for diagnostic pathology in various fields including tumor diagnosis, infectious diseases, and inflammatory diseases. Very recently, in situ hybridization (ISH) to detect tissue nucleotides by molecular technique also has been a tool of the pathological diagnosis in various diseases. This paper describes electron microscopic basis of these techniques applied to diagnostic pathology.

As techniques for electron microscopic observations, both pre-embedding and post-embedding methods have been used. As it has been widely known, in the pre-embedding method, immunohistochemical and hybridization reactions are done before plastic embedding. Peroxidase has been frequently used as a labeling reagent. On the other hand, in the post-embedding method, immunohistochemical and hybridization reactions are performed on the ultrathin plastic sections. Colloidal gold has been used extensively as a labeling material.

\section{Electron Microscopic Basis of Immunohisto- chemistry}

When the immunohistochemistry is applied to diagnostic pathology, it can be categorized according to several aspects in the detections of tissue antigens. It includes the detection of [1] new antigens such as exogenous organisms or ectopic expressions of the antigens in the cells such as hormones, [2] increased amount of normally expressed antigens such as the so-called "tumor markers" including serum proteins or cellular markers, [3] colocaliza-

\footnotetext{
Presented in part at the workshop 4 on Application of Immunohistochemistry and In Situ Hybridization to Diagnostic pathology, July 14, 1994 as part of the program at the 4th Joint Meeting of the Japan Society of Histochemistry and Cytochemistry and the Histochemical Society, held in Maui, Hawaii, July 13 to 16, 1994.

Correspondence to: Dr. R. Yoshiyuki Osamura, Department of Pathology, Tokai University School of Medicine, Boseidai Iseharacity, Kanagawa 259-11, Japan.
}

tion of multiple antigens such as serum proteins and hormones in the individual tumor cells, [4] appearance of abonormal forms of proteins such as mutant forms or prohormones without processing, and [5] changes of distribution of the antigens in the pathologic conditions such as CEA in carcinomas. The electron microscopic localization of various antigens may give basic informations for these various aspects of applications.

In this report, among the above various applications, four examples are demonstrated: [1] increased expression; prolactin in the hyperstimulated anterior pituitary cells and c-erbB- 2 in the breast carcinomas, [2] colocalization of multiple antigens; colocalization of GH and PRL in the pituitary adenomas, calcitonin (CT) and carcinoembryonic antigen (CEA) in medullary carcinomas of the thyroid gland, [3] abnormal forms of antigens; localization of prohormones in the lung cancer cells, [4] changes in distribution; CEA in the breast cancers.

\section{Increased expression of antigens}

In the anterior pituitary gland, prolactin (PRL) secreting cells are dependent on estrogen for their function. In the unstimulated condition in female rats, PRL is localized in rough endoplasmic reticula (RER), Golgi saccules and secretory granules (SG). In the female rats stimulated with estrogen, the PRL cells become hypertrophic and immunohistochemical localization of PRL is distributed in the hypertrophic cytoplasm. By electron microscopy, PRL is demonstrated in the markedly developed RER indicating marked increase in the production of PRL [4].

In the human mammary carcinomas, about $30 \%$ of the tumor shows amplification of the oncogene c-erbB-2 gene and increased c-erbB-2 protein which has $185 \mathrm{kD}$ molecular weight. The c-erbB-2 protein is a transmembranous protein and is a receptor for growth factor. Monoclonal antibodies against external and internal domains are available for immunohistochemistry. By postembedding method, the monoclonal antibody against the external domain of c-erbB-2 protein shows the reaction product at the external surface of the cell membrane of the mammary cancer cells. It has been reported that the mammary carcinomas with increased c-erbB-2 expression bear 
worse prognosis [7].

\section{Colocalization of multiple antigens}

In the human pituitary adenomas which produce $\mathrm{GH}$, the tumors are frequently multihormonal accompanied by frequent co-production of alpha subunits and PRL. In such tumors, light microscopically, GH and PRL are frequently colocalized in the same tumor cells. In such cases, GH and PRL are colocalized in the same secretory granules by post-embedding electron microscopic method suggesting co-release of these two hormones in the same secretory granules.

In the human medullary carcinomas of the thyroid gland, the tumor cells very frequently produce calcitonin (CT) and carcinoembryonic antigen (CEA) which are elevated in serum. Immunohistochemically, CT and CEA are localized in the same tumor cells. By electron microscopic post-embedding method, CT is exclusively localized in the secretory granules suggesting regulated pathways, and CEA is localized in the cell membranes and secretory vesicles suggesting constitutive pathways. They are not the antigens which demonstrate colocalization and different secretory pathways have been proposed [2].

\section{Abnormal forms of antigens}

In physiologic condition, proopiomelanocortin (POMC) is processed by proteolytic digestion and results in the formation of smaller fragments such as ACTH, MSH and endorphins. This processing is considered to occur in the secretory granules. In ectopic production of ACTH by human cancers, immunological detection demonstrated "big form" of ACTH in the serum of patients. In small cell carcinoma of the lung which is associated with Cushing syndrome, the tumor cells are focally positive for ACTH. In the carcinoma cells, secretory granules are very rare. RER are increased and prominent instead. By electron microscopic pre-embedding method, ACTH-like immunoreactivity (which include POMC) is present in well developed RER suggesting constitutive secretion of POMC. Immunoblotting demonstrates larger molecules of ACTH-like immunoreactive substance which corresponds to POMC. This indicates that prohormones are detected as abnormal forms in the cancer cells which are equiped with immature organelles for processing the physiologic secretory pathways [5].

\section{Changes of distribution}

Carcinoembryonic antigen (CEA), a glycoprotein with the molecular weight of $180 \mathrm{kD}$, is a well known tumor marker for the carcinomas of the colon, stomach, breast and others. In the breast, non-neoplastic mammary ducts show the presence of CEA in the cell membrane only at the apical portion by immunohistochemistry. In mammary cancers which produce CEA, CEA is localized, by electron microscopic pre-embedding method, in the entire cell membrane including basolateral membrane suggesting the loss of polarity in secretory pathways. This also may pay a role in the elevated CEA in the serum in the cancers which produce CEA [9].

\section{Electron Microscopic Basis for in situ Hybridization}

In situ hybridization (ISH) is a method which detects specific nucleotide sequences by the specific reaction between base pairs of nucleotides. Recently, two major advances warrant emphasis here: oligonucleotide synthesis and non-radioisotopic labeling. The oligonucleotides include various advantages over cDNA such as non-complicated synthesis and designing of their specificity and reactivity.

ISH has been utilized in two major aspects of diagnostic pathology: detection of organisms such as viruses and detection of mRNA.

\section{Detection of organisms by ISH}

For the detection of organisms, ISH is suitable especially for viruses where multiple subtypes exist such as human papilloma virus (HPV). There are several commercially available ISH staining kits for HPV, cytomegalovirus, chlamydia and others $[1,8]$.

The HPV, which is a DNA virus, has been known to consist of more than 70 subtypes. They can cause anogenital lesions. HPV subtypes 6 and 11 are well known to be related with condyloma acuminatum which is a completely benign lesion. On the other hand, subtypes 16,18 and subtypes $31,33,51$ are related with dysplasia and carcinoma of the uterine cervix. By electron microscopic preembedding method after ISH reaction on paraffin sections, HPV6/11 in the condyloma acuminatum is localized in the nuclei as virus particles suggesting the presence as plasmids. On the other hand, HPV16/18 in the cervical squamous cell carcinoma is present as integrated in the chromosomes suggesting their role in carcinogenesis [8].

\section{Detection of $m R N A$ by ISH}

By light microscopic ISH, the rat anterior pituitary gland shows many cells which contain GHmRNA and PRLmRNA diffusely in the cytoplasm. This indicates that many cells produce $\mathrm{GH}$ and PRL in the anterior pituitary gland. By electron microscopic ISH, GHmRNA is localized diffusely in the polysomes on RER suggesting widely distributed signal recognition sites on RER. For PRLmRNA, by electron microscopy, it is localized focally in the polysomes on RER suggesting selective signal recognition sites on RER for PRL. Our study is the first report on the different distribution of mRNA for different hormones in the cells [3]. Observations by ISH is important to analyze the productive activity of the cells.

\section{Conclusions}

These electron microscopic observations are expected to provide basic informations for a better understanding of 
the applications of these IHC and ISH techniques for diagnostic pathology.

\section{References}

1. Hori, S., Kawai, K., Tsutsumi, Y. and Osamura, R. Y.: Ultrastructural demonstration of Chlamydia trachomatis DNA by in situ hybridisation using a biotinylatd DNA probe, in comparison with immunoelectron microscopy. Med. Sci. Res. 19; 429-430, 1991.

2. Inada, K., Osamura, Y., Utsunomiya, H., Oda, K., Watanabe, K., Kakudo, K., Bessho, T. and Yoneyama, K.: Immunoelectron microscopical analyses of two intracellular protein secretion (transport) pathways, regulated and constitutive pathways, using thyroid medullary carcinoma. Acta Histochem. Cytochem. 26; 499, 1993.

3. Matsuno, A. and Osamura, R. Y.: Electron microscopic demonstration of GH and PRL mRNA in rat pituitary gland by in situ hybridization using non-radioistopic biotin-labeled oligonucleotide probes. Histochemistry, in press.

4. Osamura, R.Y. and Watanabe, K.: Ultrastructural localization of prolactin in estrogen-induced prolactinoma of the rat pituitary: Expremental models for the human prolactiomas and the effects of bromocriptine. Acta Pathol. Jpn 36; 1131-1137, 1986.

5. Osamura, R. Y., Tsutsumi, Y. and Watanabe, K.: Light and electron microscopic localization of ACTH and proopiomelanocrotin-derived peptides in human developmental and neoplastic cells. J. Histochem. Cytochem. 30; 919-925, 1984.

6. Sanno, N. Teramoto, A., Matsuno, A., Inada, K., Itoh, J. and Osamura, Y.: Clinical and Immunohistochemical studies on TSH-secreting pituitary adenomas: Its Multihormonality and Expression. Modern Pathology 9; 893-899, 1994.

7. Tauchi, K. and Osamura, R. Y.: Immunohistochemical localization of c-erbB-2 in human mammary carcinomas. Virchows Archiv. 416; 65-73, 1989.

8. Tsutsumi, Y., Kawai, K., Hori, S. and Osamura R. Y.: Ultrastructural visualization of human papillomavirus DNA in verrucous and precancerous squamous lesions. Acta Pathol. Jpn 41; 757-762, 1991.

9. Yamamoto, Y. and Osamura, R. Y.: Ultrastructural localization of carcinoembryonic antigen (CEA) in mammary carcinomas-Special reference on Acta Cytol. 29; 257-261, 1985. 